\title{
Interference processes in monkey auditory list memory
}

\author{
ANTHONY A. WRIGHT \\ University of Texas Health Science Center, Houston, Texas \\ and \\ HENRY L. ROEDIGER III \\ Washington University, St. Louis, Missouri
}

\begin{abstract}
A rhesus monkey's memory was tested for single items and four-item lists of natural and environmental sounds. Memory items were presented from a center speaker, followed by a retention delay and then a choice response to a test sound presented simultaneously from two side speakers. Recognition of the last item of four-item lists was much poorer than that of single items at 0-, 1-, and 2-sec delays, despite there being the same temporal relations between study and test. This result showed that the first three items proactively interfered with memory of the last list item. Proactive interference dissipated after $2 \mathrm{sec}$, revealing a recency effect that eventually equaled single-item performance. Recognition of the first item of four-item lists was much poorer than single items at 20 - and 30-sec delays, showing that the last three items retroactively interfered with memory of the first list item. The results point to the critical nature of interference processes in the understanding of serial position functions.
\end{abstract}

This article addresses two venerable issues in the study of memory. One is the shape of the bow-shaped serial position curve and how to explain it (e.g., Nipher, 1876; Ward, 1937). The second issue is the waxing and waning of interference processes in memory over time (Barnes \& Underwood, 1959; Melton \& von Lackum, 1941). We suggest that these phenomena are related and that processes of interference constitute the major explanation of the serial position curve in the study presented in this article. Of course, we are not the first to propose that interference processes account for the serial position curve (Foucault, 1928; Hull, 1935), but unlike investigators before us, we have developed tests for these interference processes (Wright, 1999a). The experiment reported in this article, we believe, provides the most compelling evidence to date that interference within a list determines the shape of the serial position function (SPF).

Proactive interference (PI) refers to the negative effects of prior learning on the retention of material learned later; retroactive interference (RI) consists of the debilitating effects of later learning on the retention of material learned earlier (see Crowder, 1976, Chap. 8, for a comprehensive

This research was supported by PHS Grant DA-10715. The authors gratefully acknowledge the careful assistance of Jacquelyne J. Rivera in training and testing this monkey. Correspondence may be addressed to A. A. Wright, University of Texas Medical School at Houston, Department of Neurobiology and Anatomy, P.O. Box 20708, Houston, TX 77225 (e-mail: Anthony.A.Wright@uth.tmc.edu),or to H. L. Roediger III, Department of Psychology, Washington University, Box 1125, One Brookings Drive, St. Louis, MO 63130-4899 (e-mail: roediger@ artsci.wustl.edu). review). The interference caused by RI has traditionallybeen conceived as akin to extinction in Pavlovian conditioning, complete with spontaneous recovery of inhibited responses (e.g., Postman, Stark, \& Fraser, 1968; Underwood, 1948a, 1948b). Paired-associate list-learning experiments (the socalled A-B, A-D procedure) have shown that RI exerts a stronger influence than PI does immediately after learning; however, with the passage of time, the influence of PI increases (Crowder, 1976, pp. 229-241). Therefore, the extinguished responses are thought to recover over time, and this recovery of the inhibited responses leads to a build-up in PI (e.g., Koppenaal, 1963; Wheeler, 1995). This pattern of interference, we believe, may be responsible for changes in SPFs following visual presentation of material in a recognition paradigm with humans, rhesus monkeys, capuchin monkeys, and pigeons (Wright, 1999b; Wright, Santiago, Sands, Kendrick, \& Cook, 1985), although tests of such interference processes have not been conducted.

Tests of monkey auditory memory originated from the desire to determine whether or not the pattern of SPF changes seen with visual memory was representative of memory generally. In the basic procedure, on each trial, the monkey hears four sounds presented from a central speaker in the test chamber. After a retention delay, a test sound is presented from two speakers to the left and the right of the monkey, and it touches the right speaker if the sound matches one of the sounds played earlier and touches the left speaker if the sound does not match any of the earlier stimuli. This general item recognition paradigm requires considerable training to achieve accurate performance.

The resulting auditory SPFs and their pattern of changes over time were the opposite of those from the classic liter- 
ature on visual memory with a variety of species (Wright, 1999a). On tests immediately after presentation of the list, the SPF showed pure primacy, with best performance on the first item and worst on the fourth item. This finding of worst recognition on the last list item, occurring just prior to the test item, is odd and counterintuitive but has been replicated in 12 independentexperiments, including the present experiment (see Wright, 1998b, 1999a, 2002). As the retention interval was increased to around $10 \mathrm{sec}$, the SPF became more typical in shape-that is, a bow-shaped curve with primacy and recency effects. At 20- and 30-sec retention delays, the SPF reflected pure recency - performance was best on the last item and worst on the first. At the very least, these remarkable changes in the serial position curve over $30 \mathrm{sec}$ of delay argue that some dynamic process (or set of processes) gives rise to the SPF and its changing shape.

We propose that the shifting balance of PI and RI over time is responsible for these changes in the rhesus monkey's auditory SPFs. Such an interference account of the monkey's auditory SPFs requires very different patterns of interference processes from those described for the classic visual SPFs. The switch from a primacy-dominated function to a recency-dominated function is remarkable, because recognition of the fourth (last) item in the sequence shows a dramatic increase over the rather brief retention intervals used. Such recovery of information strongly implicates the presence of interference and spontaneous recovery. The pattern of changes in the auditory SPF may be attributable to a decrease in PI and a concomitant increase in RI. Wright (1999a) conducted four experiments related to this proposal. Interference within lists was manipulated in two experiments by varying list item separation, and interference across lists was manipulated in two experiments by repeating items in different lists. The results strongly supported the proposal that PI affects retention immediately, with a shift to RI after a moderate retention delay.

The experiment reported in this article continued this line of research by exploring interference processes in monkey auditory memory even more directly. This experiment departed from the previous interference studies. Instead of manipulating or separating the list items, we eliminated the first three items and compared single-item memory performance with four-item list memory performance. This single-item control condition permitted us to infer whether performance changes were due simply to some process correlated with the sheer passage of time or were directly due to interference from other items presented in the list. Our predictions can be best understood by considering the immediate ( 0 -sec delay) memory test. If the first three list items interfered (proactively) with the monkey's memory of the last item, then eliminating the first three items should eliminate PI and elevate performance for the remaining list item. Single-item performance can then be compared to fourth-item performance at all retention delays, because timing parameters were held constant. If PI from the first items of the list dissipates with retention in- terval, as our proposal predicts, then fourth-item performance from four-item lists should rise with retention interval and, eventually, equal single-item performance. Thus, single-item performance serves as a control baseline or non-interference condition.

\section{METHOD}

\section{Subject}

The subject, BW, was an 18-year-old male rhesus monkey (Macaca mulatta). ${ }^{1}$ He was maintained in state- and federalapproved facilities and was fed and watered approximately $2 \mathrm{~h}$ after experimental sessions, which were conducted 5 or 6 days per week. On nonexperimental days, food (monkey chow) and water were, for the most part, unrestricted, and his diet was supplemented with fresh fruits and vegetables. He had extensive training in the auditory list memory task prior to our conducting the present experiment. Extensive training is always essential, because this is a very difficult task for monkeys to learn and perform reliably.

\section{Apparatus}

The test chamber was a modified primate cage, $58.4 \mathrm{~cm}$ wide, $58.4 \mathrm{~cm}$ deep, and $77.5 \mathrm{~cm}$ high (inside dimensions). It was located in a room with sound-deadening material on the walls. Three cage bars were removed to allow easy access $(6.7 \times 20.3 \mathrm{~cm})$ for the monkey to touch copper screens $(10.2-\mathrm{cm}$ diameter) in front of the speakers. Touching the sound sources is critical to learning behavioral tasks with auditory stimuli (cf. Harrison, Iversen, \& Pratt, 1977).

Three speakers were located outside of the cage, as is shown in the schematic in Figure 1. The center speaker was centered on the cage wall opposite the door and was $47 \mathrm{~cm}$ from the cage floor. The side speakers were located $35.6 \mathrm{~cm}$ from the center-speaker wall and $47 \mathrm{~cm}$ from the cage floor. Tubes for dispensing Tang orange drink were located on each sidewall. The tube on the right wall was $5.1 \mathrm{~cm}$ below and $5.1 \mathrm{~cm}$ to the right of the center of the right-speaker touch screen. The tube on the left wall was $5.1 \mathrm{~cm}$ below and $5.1 \mathrm{~cm}$ to the left of the center of the left-speaker touch screen. Touches were electrically monitored with high-impedance interfaces connected to a computer that controlled the experiment and collected the data.

\section{Procedure}

Trials began with presentation of a single sound or a list of four sounds from the center speaker. In the four-item list condition, each sound was presented for $2 \mathrm{sec}$, with a 1-sec interval between sounds. After presentation of a single sound or a list of four sounds, there was a retention interval $(0,1,2,10,20$, or $30 \mathrm{sec})$, and then a test sound was presented simultaneously from both side speakers. It is important to emphasize that the same sound was always presented simultaneously from both side speakers, never different sounds at the same time. The retention interval was pseudorandomly selected, with the restriction that each retention interval would be tested daily on one same and one different trial. The test sound was presented for 2-6 sec. A choice response was accepted after $2 \mathrm{sec}$, and the choice response terminated the test sound. If the test sound matched one of the list sounds (same), a touch to the right-side speaker produced $3.5 \mathrm{ml}$ of Tang orange drink from the right juice spout. If the test sound matched none of the list sounds (different), a touch to the leftside speaker produced a similar reward from the left juice spout. Failures to make a choice during the 2 to 6-sec response period or an incorrect choice resulted in a 30 -sec time-out and no reward.

An intertrial interval (ITI) followed reward or time-outs. The ITI varied quasirandomly from 12 to $27 \mathrm{sec}$ in 1 -sec steps and was counterbalanced over session blocks. The ITI was varied so that there would not be a time cue for the beginning of a trial. Tests of singleitem and four-item list memory were conducted in separate $12-$ to 

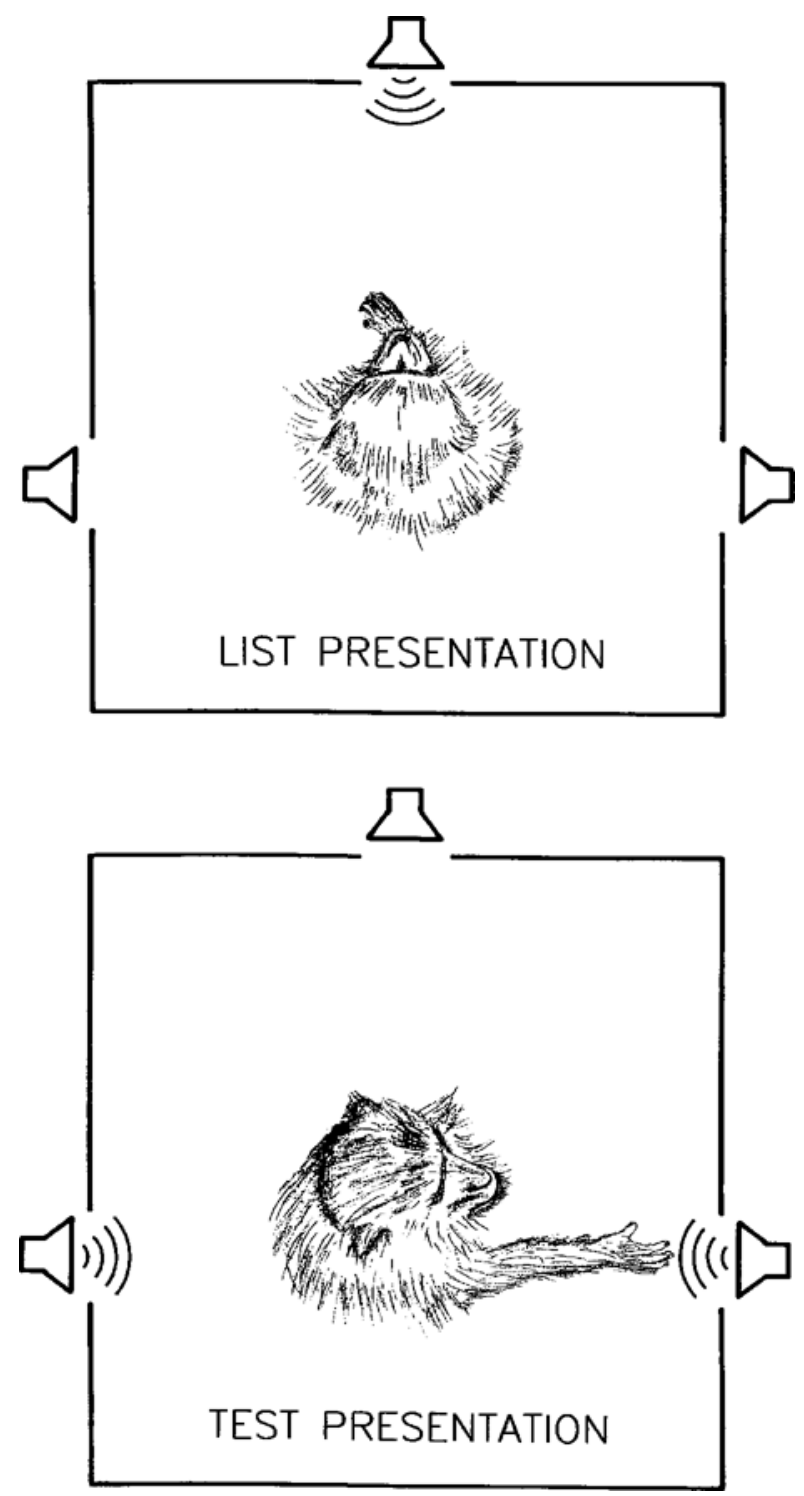

Figure 1. Schematic of the auditory testing apparatus. Upper panel: top view of the monkey being presented with an individual sound or a list of four sounds from a center speaker. Lower panel: top view of the monkey touching a right-side speaker, with a test sound being presented simultaneously from both side speakers following memory item(s) presentation(s) from the center speaker. A touch to the right-side speaker was correct when the test matched a memory item, and a touch to the left-side speaker was correct when the test did not match a memory item.

24-trial sessions, and these different test sessions were intermixed quasirandomly. All retention intervals were tested each session. Tests of the four serial positions at the six retention intervals were counterbalanced over blocks of sessions. There were 32 total tests (16 same and 16 different) of single-item memory at each retention delay, and 50 total tests of each of the four serial positions at each retention delay.

Sounds heard on any trial were not heard again for several days. The sounds were randomly selected (without replacement) from a pool of 520 sounds. The sequences of same and different trials varied daily. The natural and environmental sounds were computer dig- itized and controlled selections from 13 records (Elektra Records, New York) used by radio disc jockeys for sound effects (e.g., fog horn, mourning dove coo, dog barking, cloth tearing, etc.; for more examples, see Wright, 1998a, 1998b, 1999a, 2002).

\section{Results}

The results from the experiment are displayed in Figure 2. The upper panels show the results from single-item tests, and the lower panels show the results from four-item tests. Single-item performance (same and different) was roughly constant across delays, with good accuracy even at the long 20- and 30-sec delays. SPFs for the four-item lists showed systematic changes with retention interval. At the short delays, there was a strong primacy effect that began to dissipate at the 10-sec delay and was totally absent at longer delays. The recency effect changed in the opposite way. There was no recency effect initially, but it appeared at the 10-sec delay and became very strong later. These dynamic changes in the SPF with retention delay replicate the results previously shown for this and another monkey (Wright, 1998b, 1999a, 2002; see also Wright, 1998a). The different-trial performance was relatively stable and accurate across delays.

A major purpose of this experiment was to compare single-item and fourth-item performances. The singleitem condition was like the four-item condition without the first three list items. Figure 3 shows fourth-item performance compared to single-item performance (mean of same and different performance). Single-item performance was much more accurate than fourth-item performance over the shortest $(0,1$, and $2 \mathrm{sec})$ three retention delays. This difference was confirmed by a two-way analysis of variance (ANOVA) across delays, revealing a significant condition $\times$ delay interaction $[F(5,72)=3.1, p<.02] .{ }^{2}$ This difference was also confirmed by a one-way ANOVA on the pooled results for the three shortest delays, which showed a significant difference $[F(1,40)=32.2, p<.001]$. By contrast, performance was comparable between the two conditions at the three longest delays and was confirmed by a one-way ANOVA on the pooled results for the three longest delays $[F(1,40)=0.8, p>.6]$.

This large performance difference at short delays demonstrates the considerable effect of PI at these delays; the first three items interfered with the monkey's memory of the fourth item of the list at these short delays. We think this is a compelling conclusion; otherwise, fourth-item performance would be just like single-item performance. Fourth-item performance did rise and become equivalent to single-item performance at the longest retention intervals, demonstrating that PI from the first three items had largely dissipated by this time. PI at the short delays, we believe, was a major factor contributing to the shape of these SPFs.

Another process contributing to the shape of these SPFs was RI, which functioned primarily at the long retention intervals and adversely affected the monkey's memory for the first list items. Figure 4 shows performance for the first item of the list, compared to the single-item performance (mean of same and different) across delays. In this 

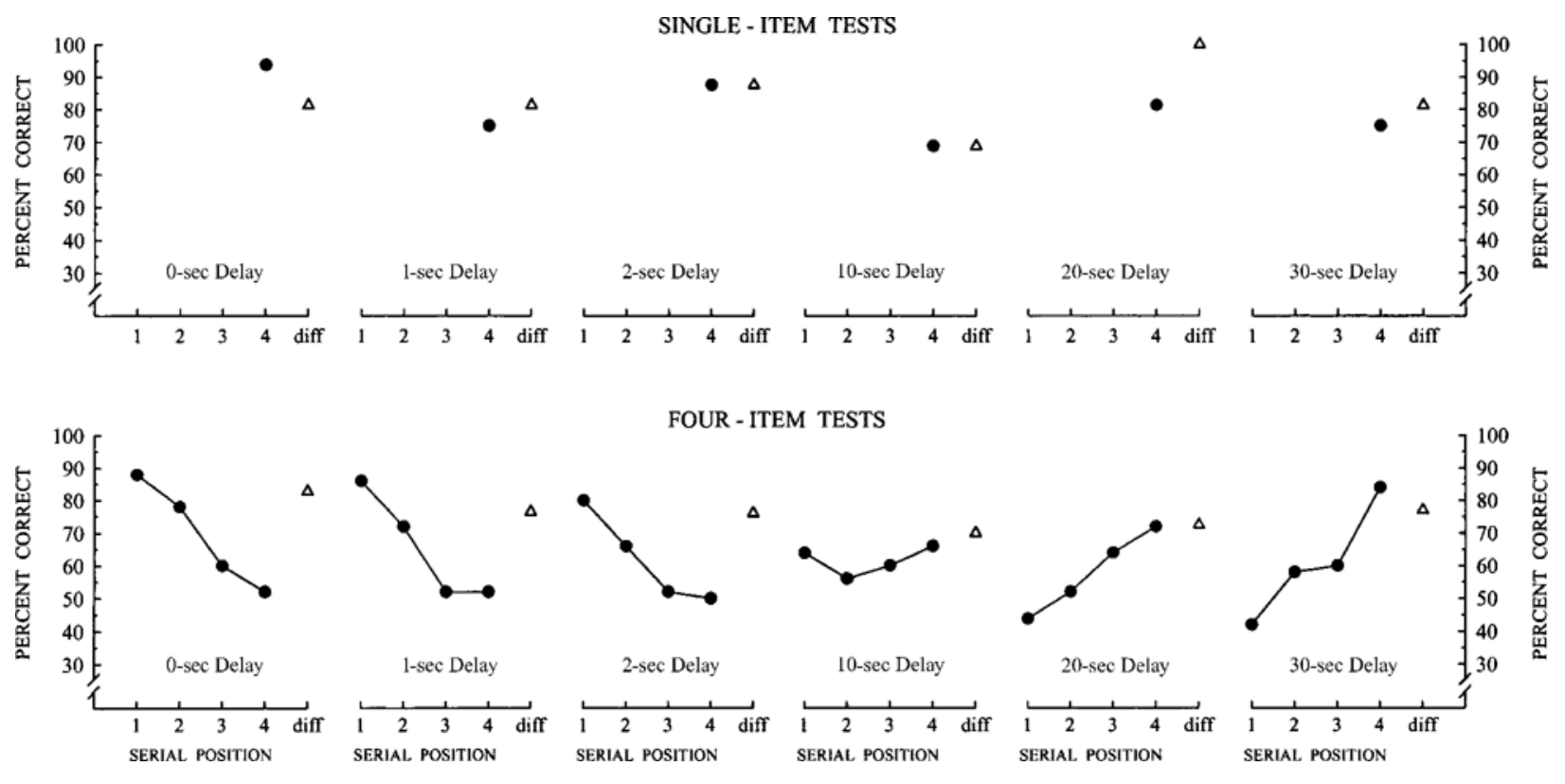

Figure 2. Upper panel: memory performance for single sounds. Lower panel: memory performance for lists of four sounds. Triangles show performance on trials (diff) in which the test did not match a memory item. Delay is the retention delay in seconds.

case, single-item performance was much more accurate than first-item performance over the longest (20- and 30sec) retention intervals. This difference was confirmed by a two-way ANOVA across delays, which revealed a significant condition $\times$ delay interaction $[F(5,72)=5.1, p<$ $.001]$. This difference was also confirmed by a one-way ANOVA on the pooled results for the two longest delays, showing a significant difference $[F(1,26)=24.2, p<$ $.0001]$. By contrast, performance was comparable between the two conditions at shorter delays $(0,1,2$, and $10 \mathrm{sec})$, confirmed by a one-way ANOVA on these pooled results $[F(1,54)=0.04, p>.8]$.

This large performance difference at the longer delays demonstrates the considerable effect of RI at these delays. It should be pointed out that this comparison is not as precise as that to fourth list item performance, because there was an added delay of the remainder of the list items for the first list item comparison. Nevertheless, the effects of RI are apparent. The last three items retroactively interfered with the monkey's memory for the first list item. This must be; otherwise, its performance would be just like it was with the single item. The equivalent performance for the first list item and the single item at the shortest retention intervals demonstrates that RI from the last three items is largely absent at these short retention delays. RI at the long delays is, we believe, a second process to explain the shape of these SPFs. At long delays, RI from the last list items eliminates the primacy effects of the SPFs.

\section{DISCUSSION}

The results from the experiment of this article were clear-cut. Performance with the single-item control did not systematically vary across the retention delays. By contrast, performance for the last item of the four-item list was much less than that for the single item at delays of 0 , 1 , and $2 \mathrm{sec}$, despite there being the same temporal relations between study and test (see Figure 3 ). The cause of this difference must be the presence of the three preceding items, which interfered proactively with memory for the last list item. This is direct evidence that PI is a controlling variable in producing the shape of these SPFs.

The time course of PI dissipation was on the order of $10 \mathrm{sec}$ or so. At this point, memory for the last list items improved, and a recency effect emerged. The recency effect increased with further retention delay. For the longest retention delays (20 and $30 \mathrm{sec}$ ), recognition of the single item was dramatically better than recognition of the first list item in the four-item list (see Figure 4). This outcome shows that RI from the last three items interfered with the monkey's memory for the first list item.

Our interference account of monkey auditory memory contrasts with the interference account from human pairedassociate experiments showing that RI is strongest immediately after learning but dissipates with time, whereas PI increases with time (Crowder, 1976). Our results showed the opposite trend. PI was strongest immediately after learning but dissipated rapidly with time, whereas RI grew slowly with time. One possibility for these differences is the modality of presentation. The dynamic pattern of SPF changes with delay for rhesus monkeys was opposite for visual, as opposed to auditory, presentations (see Wright, 1998a, Figure 8).

How the monkey's auditory SPFs might relate to human auditory memory is unclear. Except for modality effect studies (superior auditory vs. visual recency; see, e.g., Greene, 1992), which have used much longer lists, there have been few human auditory SPFs with which to make 


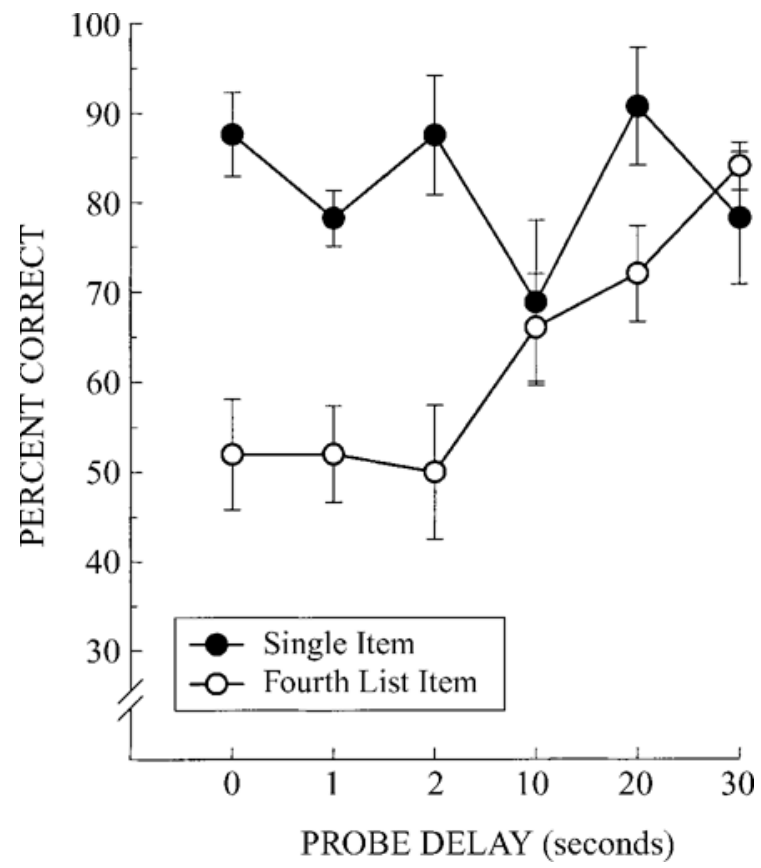

Figure 3. Single-item performance compared to last (no. 4) list item performance for the six retention delays shown in Figure 2. Error bars are standard errors of the mean.

comparisons. Long lists do not allow assessment of early SPF changes, because any initial SPF changes, such as those shown here, will have long run their course before the list is finished. In one human auditory memory experiment with short four-item lists, Knoedler, Hellwig, and Neath (1999, Experiment 3) tested 1- and 3-sec retention delays and found a pattern of SPF changes that resembled human (and animal) visual SPFs, but not monkey auditory SPFs. The problem with interpreting these results is that visual and auditory memory may have been confounded. "Auditory" items were actually visually presented (written) on a computer screen (but were pronounced to make them "auditory") and were names of Snodgrass and Vanderwart's (1980) objects that could easily be (visually) imagined. If the subjects were visually, as well as auditorily, coding these items, then it would be no surprise to find SPFs that resembled visual SPFs. Another auditory experiment used lists of five different tones (Surprenant, 2001). Tone lists were presented in pairs, and the two lists were identical on half the trials. On the other half of the trials, one tone in the second list (position varied) was different from that on the first list. The participants judged whether the two lists were the same or different. The effect of testing recognition with an entire second list, as opposed to a single item from the first list, is unclear, but this procedure, along with using a list of musical notes, may have encouraged the subjects to configure the list as a whole (e.g., a tune), rather than process the items as five independent memory items. Since the issue here is the shape of the SPF with single-item recognition tests, it seems important to have independent memory items. Nevertheless, the results from this experiment showed that recency weakened and primacy strengthened for 2-sec delay SPFs, as opposed to 0 -sec delay SPFs - a result similar to visual SPFs.

One possibility is that rhesus monkey and human auditory memory are fundamentally different, but we think that this possibility is unlikely, given the extensive functional similarities between rhesus monkeys and humans from other studies. A more likely possibility, we feel, is that monkey memory is fundamentally similar to human memory but is stripped of the strategic processing humans regularly employ in tasks and tests. One of us (the first author) has found (in unpublished pilot experiments) that it is much more difficult to test "pure" human auditory memory than "pure" visual memory (e.g., with kaleidoscope patterns). Unlike visual items, auditory items "unfold" in time, and subjects have a tendency to fragment the stimulus and to code distinctive features. Distinctive feature coding can take the form of "visual" coding even with auditory stimuli (e.g., Campbell \& Dodd, 1980). If visual coding were to occur, it should be no surprise to find "auditory" SPFs that resemble visual SPFs. These considerations are admittedly somewhat speculative and deserve careful experimental study.

A related and also speculative issue is the following often raised question: What possible (evolutionary) value could there be for opposite-shaped auditory and visual SPFs? If humans were to have opposite-shaped SPFs, then bimodal coding of items would enhance memory much more than redundant same-modality coding would, because auditory memory would be good when visual memory was poor and vice versa. Although animals are less

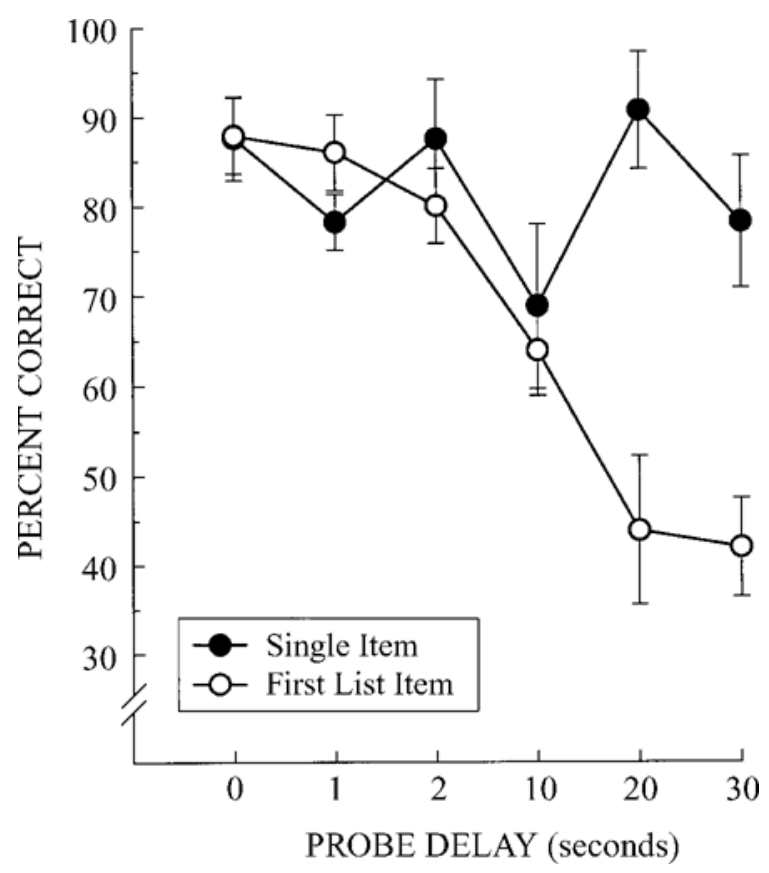

Figure 4. Single-item performance compared to the first (no.1) list item performance for the six retention delays shown in Figure 2. Error bars are standard errors of the mean. 
likely to code items than are humans, associative learning studies with animals have shown that visual stimuli are better associated with food and auditory stimuli with danger than the other way around (e.g., Shapiro, Jacobs, \& LoLordo, 1980). Consider visual memory. If an animal had luck foraging for food in one patch (e.g., a pine tree), then it will need to remember (visual recency, short delay) to go to a similar patch (i.e., another pine tree) to continue feeding. If, on the other hand, an animal fed in a diurnally depleting patch (e.g., berries that ripen in the morning), then it will need to remember (visual primacy, long delay) to go to this same patch first thing the next morning. For auditory memory, if an animal heard a danger sound, then it will need to remember (auditory primacy, short delay) the starting point of the sound to determine whether the sound (e.g., one by a predator in the forest) is coming toward it or going away from it. If, on the other hand, an animal heard a danger sound and the sound stops, then it will need to remember (auditory recency, long delay) where the sound stopped to avoid the spot where the predator might be hiding in wait.

There have been, to be sure, accounts other than interference of the serial position function. The popular and venerable dual-store models claim that the recency effect represents short-term memory (STM) and decays with time (e.g., Atkinson \& Shiffrin, 1968; Gillund \& Shiffrin, 1984; Haarmann \& Usher, 2001). But the monkey's auditory recency effect cannot represent STM, because a recency effect is not present initially and its later appearance is more like an LTM effect than an STM effect. Other accounts of dynamically changing SPFs have included temporal distinctiveness (e.g., Knoedler et al., 1999; Neath, 1993; Neath \& Knoedler, 1994) and storage versus retrieval strength (Bjork, 2001). An item's temporal distinctiveness is thought to change with time, like a receding row of telephone poles; those in the distance are individually less distinct than those close by (Crowder \& Neath, 1991). But as Bjork has pointed out, temporal distinctiveness cannot account for absolute (as opposed to relative) memory recovery, as shown by the visual primacy effect. To account for absolute recovery, Bjork proposed a storage strength versus retrieval strength model. However, Bjork's model depends on repeated learning of individual memory lists and would not apply to absolute recovery following single presentations of lists, like those used to test the monkey's auditory (and visual) memory.

The view presented in this article is that retrieval failure underlying the SPF is the result of interference. Our experiments presented here and elsewhere (e.g., Wright, 1999a) have shown that, for rhesus monkeys, PI within an auditory memory list causes retrieval failure of the last list items. Release from this (proactive) interference causes an absolute increase in recency, and the resulting (retroactive) interference causes retrieval failure for the first items of the list. With regards to the auditory memory results presented in this article and elsewhere, no explanation fares as well as interference. A critic might complain that invoking processes of PI and RI may only name, rather than explain, our findings. However, we directly manipulated interference in the experiment in the present article, and the results uniquely support our account.

Our account is further bolstered by the results from four other experiments with this and another monkey (Wright, 1999a). In two of these experiments, list item separation was manipulated (increased), and the main finding was that PI from the first list items at short retention delays was reduced and produced improved recency (as much as $44 \%$ improved recency). In the other two experiments, some or all of the stimuli were selected from a small eightitem set, and this manipulation increased interference across (as opposed to within) lists. In one case, recency improved (54\%) when first-item performance (and PI) was reduced at 0 -sec delays. In another case, primacy improved (also 54\%) when last-item performance (and RI) was reduced at 20 -sec delays. Together the results from these four experiments, plus the experiment of the present article, converge on the conclusion that PI is instrumental in producing the short-delay all-primacy auditory SPFs and that RI is instrumental in producing the long-delay allrecency auditory SPFs.

In conclusion, we have provided evidence for interference being instrumental in the changes in the SPF for auditory lists in the item recognition paradigm. These results show that PI is strong just after an auditory list is presented, thereby depressing recognition of the last items. Over time, PI weakens, and RI strengthens, for these auditory lists. After $30 \mathrm{sec}$, the shape of the SPF has changed dramatically. The results presented in this article show that interference from members of a list produces a dynamically changing pattern of SPFs. Although this is a descriptive, rather than a quantitative, model, the evidence indicates that interference is the root cause of the SPF changes. As far as we can tell, there is no viable rival theory. For example, one can cast distinctiveness as being a result of interference, but not the other way around. We cannot think of a way in which distinctiveness could increase with delay and account for the absolute performance increases (auditory recency or visual primacy) or a reason why distinctiveness of auditory and visual lists would be opposite in their properties for monkeys and humans. We feel that the complex patterns of serial position effects and their changes reported here and elsewhere (Wright, 1998a, 1998b, and 1999a) provide a promising target for memory theories purporting to deal with serial order and serial position effects.

\section{REFERENCES}

Anderson, N. H. (2002). Methodology and statistics in single-subject experiments. In H. Pashler \& J. Wixted (Eds.), Stevens' Handbook of experimental psychology (pp. 301-337). New York: Wiley.

Atkinson, R. C., \& Shiffrin, R. M. (1968).Human memory: A proposed system and its control processes. In K. W. Spence \& J. T. Spence (Eds.), The psychology of learning and motivation (Vol. 2, pp. 89-105). New York: Academic Press.

Barnes, J. M., \& Underwood, B. J. (1959). "Fate" of first-list associations in transfer theory. Journal of Experimental Psychology, 58, 97105 . 
BJork, R. A. (2001). Recency and recovery in human memory. In H. L. Roediger III, J. S. Nairne, I. Neath, \& A. M. Surprenant(Eds.), The nature of remembering: Essays in honor of Robert G. Crowder (pp. 211232). Washington, DC: American Psychological Association.

CAmpbell, R., \& DodD, B. (1980). Hearing by eye. Quarterly Journal of Experimental Psychology, 32, 85-99.

Crowder, R. G. (1976). Principles of learning and memory. Hillsdale, NJ: Erlbaum.

Crowder, R. G., \& Neath, I. (1991). The microscope metaphor in human memory. In W. E. Hockley \& S. Lewandowsky (Eds.), Relating theory and data: Essays on human memory in honor of Bennet B. Murdock, Jr. (pp. 111-125). Hillsdale, NJ: Erlbaum.

Foucault, M. (1928). Les inhibitions internes de fixation. Anneé Psychologique, 29, 92-112.

Gillund, G., \& Shiffrin, R. M. (1984). A retrieval model for both recognition and recall. Psychological Review, 91, 1-67.

Greene, R. L. (1992). Human memory: Paradigms and paradoxes. Hillsdale, NJ: Erlbaum.

HAARmann, H., \& Usher, M. (2001). Maintenance of semantic information in capacity-limited item short-term memory. Psychonomic Bulletin \& Review, 8, 568-578.

Harrison, J. M., Iversen, S. D., \& Pratt, S. R. (1977). Control of responding by location of auditory stimuli: Adjacency of sound and response. Journal of the Experimental Analysis of Behavior, 28, 243251.

Hull, C. L. (1935). The conflicting psychologies of learning-a way out. Psychological Review, 42, 491-516.

Knoedler, A. J., Hellwig, K. A., \& Neath, I. (1999). The shift from recency to primacy with increasing delay. Journal of Experimental Psychology: Learning, Memory, \& Cognition, 25, 474-487.

KoppenaAL, R. J. (1963). Time changes in the strengths of A-B, A-C lists: Spontaneous recovery? Journal of Verbal Learning \& Verbal Behavior, 2, 310-319.

Melton, A. W., \& von Lackum, W. J. (1941). Retroactive and proactive inhibition in retention: Evidence for a two-factor theory of retroactive inhibition. American Journal of Psychology, 54, 157-173.

Neath, I. (1993). Distinctiveness and serial position effects in recognition. Memory \& Cognition, 21, 689-698.

Neath, I., \& Knoedler, A. J. (1994). Distinctiveness and serial position effects in recognition and sentence processing. Journal of Memory \& Language, $\mathbf{3 3}, 776-795$.

NipHER, F. E. (1876). On the distribution of numbers written from memory. Transactions of the Academy of St. Louis, 3, 79-80.

Postman, L., Stark, K., \& Fraser, J. (1968). Temporal changes in interference. Journal of Verbal Learning \& Verbal Behavior, 7, 672-694.

Shapiro, K. L., Jacobs, W. J., \& LoLordo, V. M. (1980). Stimulusreinforcer interactions in Pavlovian conditioning of pigeons: Implications for selective associations. Animal Learning \& Behavior, 8, 586-594.

SNOdGrass, J. G., \& VANDERWART, M. (1980). A standardized set of 260 pictures: Norms for name agreement, familiarity, and visual complexity. Journal of Experimental Psychology: Learning, Memory, \& Cognition, 6, 174-215.
Surprenant, A. M. (2001). Distinctiveness and serial position effects in tonal sequences. Perception \& Psychophysics, 63, 737-745.

UNDERWOOD, B. J. (1948a). Retroactive and proactive inhibition after five and forty-eight hours. Journal of Experimental Psychology, 38, 29-38.

UNDERWOOD, B. J. (1948b). "Spontaneous recovery" of verbal associations. Journal of Experimental Psychology, 38, 429-439.

WARD, L. B. (1937). Reminiscence and rote learning. Psychological Monographs, 49(4).

WheELer, M. A. (1995). Improvement in recall over time without repeated testing: Spontaneous recovery revisited. Journal of Experimental Psychology: Learning, Memory, \& Cognition, 21, 173-184.

Wright, A. A. (1998a). Auditory and visual serial position functions obey different laws. Psychonomic Bulletin \& Review, 5, 564-584.

Wright, A. A. (1998b). Auditory list memory in rhesus monkeys. Psychological Science, 9, 91-98.

Wright, A. A. (1999a). Auditory list memory and interference in monkeys. Journal of Experimental Psychology: Animal Behavior Processes, 25, 284-296.

Wright, A. A. (1999b). Visual list memory in capuchin monkeys (Cebus apella). Journal of Comparative Psychology, 113, 74-80.

Wright, A. A. (2002). Monkey auditory list memory: Tests with mixed and blocked retention delays. Animal Learning \& Behavior, 30, 158164.

Wright, A. A., Santiago, H. C., Sands, S. F., Kendrick, D. F. \& Cook, R. G. (1985). Memory processing of serial lists by pigeons, monkeys, and people. Science, 229, 287-289.

\section{NOTES}

1. A single monkey, BW, was tested in this experiment. In prior work, another monkey, FD, had also been trained on this task and both monkeys were tested. The second monkey died before this experiment was begun. However, in prior work, both monkeys provided quite similar data.

2. For the purposes of the statistical analyses presented in this article, including the SEMs shown in Figures 3 and 4, memory performance was divided into 10 blocks of five consecutive tests at each serial position, and into 4 blocks of four consecutive tests (both same and different trials) for the single-item memory task. Although these are tests with a single subject, the experiment avoids many reliability and validity problems (e.g., serial correlation, carryover effects, etc.) typical of single-subject statistical tests (Anderson, 2002). Retention intervals were tested randomly, which tends to avoid (or average out) problems of local drift and serial correlation. Similar arguments can be applied to interleaving the fouritem and single-item tests. Finally, there was a built-in monitor to ensure that drops in accuracy were mnemonic, not attentional or motivational; SPFs at all delays had points of good accuracy (short-delay primacy or long-delay recency).

(Manuscript received January 14, 2002; revision accepted for publication June 14, 2002.) 